\title{
Prior, translational semantics, and the Barcan formula
}

\author{
B. Jack Copeland ${ }^{1,2}$
}

Received: 12 October 2015 / Accepted: 17 October 2015 / Published online: 19 December 2015

(C) Springer Science+Business Media Dordrecht 2015

\begin{abstract}
The revolution in semantics in the late 1960s and 1970s overturned an earlier competing paradigm, 'translational' semantics. I revive and defend Prior's translational semantics for modals and tense-modals. I also show how to extend Prior's propositional modal semantics to quantificational modal logic, and use the resulting semantics to formalize Prior's own counterexample to the Barcan Formula.
\end{abstract}

Keywords Prior - Davidsonian semantics - Modal semantics - Tense logical semantics $\cdot$ Substitutional quantifiers $\cdot$ Barcan formula

\section{Introduction: the translational paradigm}

Translational semantics is a nowadays neglected form of semantics that pre-dates the meteoric rise of Tarski-style truth-conditional semantics in the 1960s and 1970s. In translational semantics, the key semantical predicate is translates as, or some cognate term, rather than is true (in or at). Translational semantics for both extensional and intensional languages give accounts of the meanings of the sentences of the target language, by virtue of specifying translations of these sentences into a semantically more perspicuous language, such as an enriched predicate calculus.

A translational semantics will typically not involve semantic ascent, and will not offer the modern-day semantic staples of satisfaction clauses, valuations that supply formal truth-conditions, set-theoretic constructs constituting the semantic values of sub-sentential terms of the object-language, and so forth. A translational semantics

\footnotetext{
$\triangle \quad$ B. Jack Copeland jack.copeland@canterbury.ac.nz

1 University of Canterbury, Christchurch, New Zealand

2 University of Queensland, Brisbane, Australia
} 
may be formal, recursive, and completely rigorous. Translational semantics is, however, virtually unknown today. Max Cresswell, in his contribution to this volume, effectively sums up the modern attitude when he says: 'Semantics ... is not the same as translating an object language into another object language'. ${ }^{1}$

Translational semantics was supplanted by the new truth-conditional paradigm during a heady revolution that began in the late 1960s, and reached full force during the 1970s. Some of the precipitating events were the publication of Donald Davidson's paper 'Truth and Meaning' in 1967, Saul Kripke's succession of papers on modal semantics, beginning in 1959 and continuing throughout the 1960s, and the work of Jaakko Hintikka, David Kaplan, Richard Montague, Dana Scott, and others. In his now classic 1967 paper, Davidson urged that Tarski's recursive theory of truth be regarded as a theory of meaning, arguing that 'a theory of meaning for a language $L$ shows "how the meanings of sentences depend upon the meanings of words" if it contains a (recursive) definition of truth-in-L'. ${ }^{2}$ He added polemically: 'And, so far at least, we have no other idea how to turn the trick'. In fact, as I shall explain, translational semantics is and was perfectly capable of showing how the meanings of sentences depend on the meanings of their components.

An influential manifesto of the new paradigm in semantics was the 1976 collection Truth and Meaning, edited by two young storm troopers of the revolution, Gareth Evans and John MacDowell. Their introduction to this volume contained a spirited attack on the older rival paradigm. They said, 'We call this view, according to which the job of a theory of meaning is to provide rules relating sentences to their semantic representations, "translational semantics". 3 They intended to leave their readers in no doubt that translational semantics was utterly bankrupt, freely using words like 'wrong', 'objectionable', and 'utter incapacity', in order to express their contempt for the outgoing paradigm. Although rousing in those revolutionary days, their arguments have not withstood the test of time. For example, they exhorted 'What we ought to be doing is stating what sentences of the language mean, stating something such that, if someone knew it, he would be able to speak and understand the language'. ${ }^{4}$ However, that is exactly what translational semantics does do.

Their key objection, which they said 'devastates translational semantics', is that 'someone could know it [the semantics] without understanding the language of which it is theory'. ${ }^{5}$ Yet exactly the same is true of truth-conditional semantics. Someone could know, for example, that $v(\mathrm{~L} A, i)=\mathrm{T}$ if and only if $\forall j(j \leq i \rightarrow v(A, j)=\mathrm{T})$ without thereby understanding what the object language statement LA means. For a truth-conditional semantics to succeed in assigning meanings to the statements of the object language, the meta-language must not consist of mere formalism but must be interpreted (e.g., in terms of possible worlds, accessibility, and truth at a world), and moreover must be understood as such by the person for whom the theory is

\footnotetext{
1 Cresswell (2015, p. 1-17).

2 Davidson (1967, p. 310).

3 Evans and MacDowell (1976, p. ix).

4 Evans and MacDowell (1976, p. ix).

5 Evans and MacDowell (1976, p. x).
} 
to serve as a theory of meaning for the object language. ${ }^{6}$ Likewise with translational semantics: for the theory to serve as a theory of meaning, the semantically perspicuous language in which meaning-giving translations are formulated must - of course-be understood by the person for whom the theory is to serve as a theory of meaning; and if someone does understand the semantically perspicuous language, and does know the rules relating target sentences to their translations, then they will ipso facto also understand the statements of the target language.

Because Davidsonian truth theories are extensional, extensionally equivalent expressions can be substituted into the theory without changing the truth-values of the relevant T-sentences. Evans and MacDowell called the result of performing extensionally equivalent but intentionally inequivalent substitutions a 'mutilated' truth theory. A favourite example was a mutilated truth theory producing the true T-sentence " Snow is white" is true if and only if grass is green', together with indefinitely many other true but mutilated T-sentences. Evans and MacDowell were much exercised by the phenomenon of mutilated truth theories, concluding that these posed a severe challenge to Davidson's original conception of truth-theoretic semantics (here they were strongly influenced by arguments put forward by John Foster and Brian Loar). ${ }^{7}$ The problem, of course, is that since the mutilated truth theory does not constitute a theory of meaning for the language, how are mutilated truth theories to be excluded from the class of genuinely meaning-giving truth theories? This was the answer that Evans and MacDowell gave in 1976: "the conditions must ensure that the sentences used on the right translate those mentioned on the left' ${ }^{8}$ They pleaded against concluding 'that we have now come full circle'; and they were right, in that their semantics remained truth-conditional rather than translational. ${ }^{9}$ But one could be excused for wondering whether (despite their tendentious arguments) the truth-conditional curlicues of their theory-as in "The German sentence "Schnee ist Weiss" is true if and only if snow is white' - could not be dispensed with in favour of a recursive, purely translational semantics.

Another of the semantical revolutionaries was David Lewis, who coined the mantra Semantics with no treatment of truth conditions is not semantics. ${ }^{10}$ In two well-known paragraphs of his 1970 article 'General Semantics', Lewis attacked a then-popular form of translational semantics, namely that semantic interpretation consists of the systematic translation of sentences of the target language into an artificial language that Lewis dubbed Semantic Markerese. He urged that 'Translation into Markerese is at best a substitute for real semantics'. ${ }^{11}$ He mocked: 'Translation into Latin might serve as well, except insofar as the designers of Markerese may choose to build into it useful features [such as] freedom from ambiguity [and] grammar based on symbolic logic'. ${ }^{12}$

\footnotetext{
6 Copeland (1979, 1986).

7 Evans and MacDowell (1976, pp. xiii-xiv). Foster, J. A. 'Meaning and truth theory', and Loar, B. 'Two theories of meaning', both in Evans and MacDowell (1976).

8 Evans and MacDowell (1976, pp. xiv-xv); my emphasis.

9 Evans and MacDowell (1976, p. xv).

10 Lewis (1970, p. 18).

11 Lewis (1970, p. 18).

12 Lewis (1970, p. 19).
} 
These are, however, crucial differences. The translational semanticist certainly does not think that translation into any old language — Latin, English, Polish — is a way to do semantics. The selected language must have features that make it properly suitable for the job, and a regimented and logically transparent language based on the predicate calculus is a much more promising candidate than Latin.

Mockery aside, Lewis gave two arguments against translational semantics. The first was essentially the same as the point later emphasized by Evans and MacDowell: 'But we can know the Markerese translation of an English sentence', Lewis wrote, 'without knowing the first thing about the meaning of the English sentence: namely, the conditions under which it would be true'. ${ }^{13}$ However, what Lewis says here is simply untrue. In so far as the assertions $p$ and It is true that $p$ give the same information, the speaker who knows the English sentence's translation $p$ into the semantical language, and understands the semantical language, does thereby know the canonical conditions under which the English sentence is true.

Lewis's second argument was that, because the 'Markerese method ... deals with nothing but symbols', this 'prevents Markerese semantics from dealing with the relations between symbols and the world of non-symbols - that is, with genuinely semantic relations' ${ }^{14}$ However, the translationalist's semantical language brings its own apparatus of real-world reference, predication, quantification, and ontological commitment, and this apparatus is in gear and working when the translation-sentences are spoken or written by the understanding speaker. For example, a sentence of the translationalist's semantical language of the form ' $a$ bears $R$ to $b$ ' is no less about the objects $a$ and $b$ and the relation $R$ than is the statement that would appear on the right hand side of a truth-conditional semantics, such as 'The ordered pair consisting of the denotation of term ' $a$ ' followed by the denotation of term ' $b$ ' is a member of the extension of the relation $R$ '. When the understanding speaker knows that the translation of $S$ is $P$, then they ipso facto have knowledge of the relationship between the string of symbols $S$ and the world of non-symbols.

In his 1975 book The Language of Thought, Jerry Fodor came roaring to the defence of translational semantics, saying that 'translational theories' have 'recently become the object of considerable philosophical disapprobation, much of it, I think, quite undeserved'. ${ }^{15}$ He responded to Lewis's influential critique, saying 'It seems pertinent ... to comment on a certain unfairness that attaches to Lewis' remarks if they are taken as a general criticism of translational approaches to semantics', continuing:

It is ... true that 'we can know the Markerese translation of an English sentence without knowing the first thing about the meaning of the English sentence.' ... But, of course, this will hold for absolutely any semantic theory whatever so long as it is formulated in a symbolic system; and, of course, there is no alternative to so formulating one's theories. We're all in Sweeney's boat; we've all gotta use words when we talk. Since words are not, as it were, self-illuminating like globes on a Christmas tree, there is no way in which a semantic theory can guarantee

\footnotetext{
13 Lewis (1970, p. 18).

14 Lewis (1970, p. 19).

15 Fodor (1975, p. 119).
} 
that a given individual will find its formulae intelligible. So, the sense in which we can 'know the Markerese translation of an English sentence without knowing ... the conditions under which it would be true' is pretty uninteresting. ${ }^{16}$

Of course, it was not only philosophical arguments that led to the demise of the translational paradigm. The sheer power and elegance of truth-conditional semantics resulted in its widespread use by logicians and mathematicians. Mathematician Frank Drake, who did work in modal logic using translational semantics before Kripke's model-theoretic approach to the semantics of modal logic became the default, said:

I certainly regarded Kripke's later work as superseding mine, and even more so the work of Dana Scott which I learned of in about 1965. I can remember being glad to have my PhD in my pocket when I saw that work. ${ }^{17}$

Yet once translational semantics is absolved from the accusations of the revolutionaries, might there not be a role for it in modern logic? Translational semantics is leaner than its truth-theoretic brother. It carries no commitment to set theory, avoids semantic ascent, does not assign abstract semantic values to subsentential elements of the language, nor indeed to the sentences themselves, and approaches closer to the homophonic ideal of giving the semantics of a language without exceeding the resources of that very language.

\section{Prior and translational semantics}

Arthur Prior gave translational semantics for both tensed languages and alethic modal languages. In the case of tensed languages, an early example of his translational semantics appeared in his 1954 presidential address ${ }^{18}$ to a conference in Wellington, New Zealand, later published as 'The Syntax of Time-Distinctions'; and in the case of alethic modal languages, a mature example of his translational approach appeared in his 1962 paper 'Possible Worlds'. ${ }^{19}$

Prior never believed that the semantical languages in which he couched his translations were, as he put it, 'metaphysically fundamental', and in 'The Syntax of Time-Distinctions' he issued a warning against regarding his translations as 'a metaphysical explanation' of the tenses. ${ }^{20}$ Elsewhere, Prior gave the same warning in the case of alethic modal logic, saying:

Some writers have attempted to represent modal logic ... as a kind of disguised quantification theory - perhaps with quantification over 'possible states of affairs'. I don't myself think that this will do ... though I won't develop my objections to it now. ${ }^{21}$

\footnotetext{
16 Fodor (1975, pp. 120-121).

17 Letter from Drake to Copeland (2000); Drake (1962).

18 See Copeland (1996).

19 Prior $(1958,1962 a)$.

20 Prior (1958, p. 115).

21 Prior (1976, p. 128).
} 
He said this in his article 'What Is Logic?', in 1962, the same year that he published his own translational semantics for modal logic, itself couched in terms of quantification over possible worlds (a semantics that he developed with the Irish logician Carew Meredith). Prior should not be understood, in the above quotation, as having some objection to possible worlds semantics per se; his point was, again, the metaphysical one that the language of possible worlds, no less than a language involving quantification over times, is ultimately to be explained in terms of a language with modal operators (or, in the case of time, tense operators). This was a theme to which he returned in his final book Worlds, Times and Selves. However, despite his metaphysical orientation on these matters, he considered the translational approach involving quantification over worlds and times illuminating enough to make frequent use of these semantics.

Prior died in 1969, never witnessing the full force of the revolution against translational semantics. How, or whether, he would have contributed to the debate we shall never know, although his 1957 review of Tarski's Logic, Semantics and Metamathematics certainly contains ammunition that could have been used against the revolutionaries. For example, Prior said 'One objection which may be made against Tarski ... is that far too much is said in this context about "sentences". 22 Prior disliked semantic ascent and was much more interested in propositions than sentences. He preferred to reformulate Tarski's T-sentence

The English sentence 'Snow is white' is true if and only if snow is white.

His reformulation was the propositionalised, non-metalinguistic

Whoever says that snow is white says so truly if and only if snow is white. ${ }^{23}$

In his review of Tarski, Prior dwelt on what he called a 'defect' in Tarski's truth theory, namely that the various different truth clauses (one for negation, one for disjunction, one for the universal quantifier, and so on) produce a 'piecemeal definition of truth ... and the more complex the language considered the more pieces there will be'. The result is that " "true" has different meanings when applied to sentences of different sorts'. ${ }^{24}$ Perhaps Prior would have wielded this same point against the Davidsonian conception of Tarskian semantics: the semantics requires as many different concepts of truth as there are recursive clauses in the semantics.

To make the notion of translational semantics concrete, the next section gives some details of Prior's semantics for the languages of modal and tense logic.

\section{Prior's tense-modal and modal semantics}

In 'The Syntax of Time-Distinctions', Prior explained that his new PF-calculus isas he put it-'interpretable within' what he called the $l$-calculus, where $l$ is read 'is later than', and 'interpretable within' evidently means for Prior that formulae of the $\mathrm{PF}$-calculus can be translated systematically into the $l$-calculus. Where $\mathrm{z}$ is a variable

\footnotetext{
22 Prior (1957b, p. 406).

23 Prior (1961, p. 28).

24 Prior (1957b, pp. 408-409).
} 
representing 'the date at which the proposition under consideration is uttered', Prior's semantics for his tense operators $\mathrm{P}, \mathrm{F}, \mathrm{G}$, and $\mathrm{H}$, is as follows:

'Fq' ('It will be the case that q') is interpreted as ' $\exists x(l x z \& q x)$ '.

'Pq' ('It has been the case that q') is interpreted as ' $\exists x(l z x \& q x)$ '.

'Gq' ('It will always be the case that q') is interpreted as ' $\forall \mathrm{x}(l \mathrm{xz} \rightarrow \mathrm{qx})$ '.

'Hq' ('It has always been the case that q') is interpreted as ' $\forall \mathrm{x}(l \mathrm{zx} \rightarrow \mathrm{qx})$ '. ${ }^{25}$

For anyone who understands Prior's augmented predicate calculus, his translational clauses offer an explanation of the meanings of 'Fq', 'Pq', 'Gq', and 'Hq'. Moreover, the semantics arguably shows how the meanings of complex sentences arise from the meanings of their parts, as Davidson required of a theory of meaning. 'GPq' (Prior's example) is effectively translated in two simultaneous steps, the first addressing the meaning of the contained ' $G$ ' and the second addressing the meaning of the contained 'P', viz

$$
{ }^{\prime} \mathrm{GPq}{ }^{\prime} \Rightarrow{ }^{\circ} \forall \mathrm{x}(l \mathrm{xz} \rightarrow \mathrm{Pqx}){ }^{\prime} \Rightarrow{ }^{\prime} \forall \mathrm{x}(l \mathrm{xz} \rightarrow \exists \mathrm{y}(l \mathrm{xy} \& \mathrm{qy}))^{\prime} .
$$

Prior's translational modal semantics is formally very similar to his tense-modal semantics. The variables refer to worlds instead of times or dates, and an accessibility relation between worlds replaces the $l$-relation. In a section entitled 'The Logic of World-Accessibility' in a 1962 article, Prior wrote:

Suppose we have the usual variables p, q, r, etc., for statements, and a, b, c, etc., for names of 'worlds' or total states of affairs. Let us then write pa for 'It is the case in world a that p'. ... Let us ... interpret (Lp)a, 'Necessarily-p in world a', as short for ... ' $p$ is true in a and in all worlds accessible from it'. ${ }^{26}$

Prior provided what he called an 'interpretation' of modal statements by translating them systematically into his extended predicate calculus. This is Prior's possible worlds semantics, developed independently of Kripke. ${ }^{27}$ His notation 'pa' is read as 'It is the case in world a that p', which, as in the above quotation, he sometimes glosses as ' $p$ is true in a'. Just as in the tensed case, Prior's various translation-clauses give meanings to the modal sentences under consideration; and, again, his semantics shows how the meanings of complex sentences, such as the S4 and S5 axioms, arise recursively from the meanings of their parts. Prior said that by reference to this interpretation of modal statements, 'many features of the logic of necessity and possibility are readily explained'.

The semantics first appeared in Prior's 1956 typescript 'Interpretations of Different Modal Logics in the "Property Calculus", which I found in the archives of the University of Canterbury (see Fig. 1). ${ }^{28}$ This short typescript, which Prior circulated in mimeograph form, anticipates key elements of modern possible worlds semantics for propositional T, S4 and S5, years before Kripke's first publications appeared. To the

\footnotetext{
25 Prior (1958, pp. 112-113).

26 Prior (1962b, p. 140).

27 See Copeland (2002).

28 Meredith and Prior (1956).
} 
UNIVERSITY OF CANTERBURY.

DEPARTMENT OF PHILOSOPHY.

\section{INTERPRETATIONS OF DIFFERENT MODAL LOGICS \\ (G.A.M., August $1956 ;$ recorded \& expanded A.N.P.)}

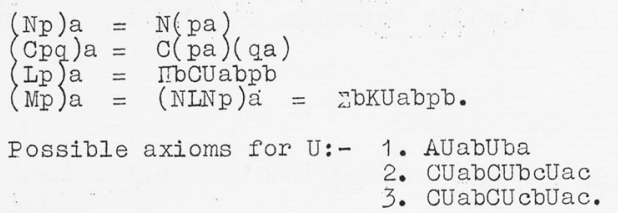

1 gives 4. Uaa

3, 4 give 5. CUabuba.

3, 5 give 2 .

Df.I and quantification theory give

6. (CLCpqCLpLq)a,

i. e. CIrbCUabCpbqb CIrbCUabpbIroCUabqb.

4 gives

7. (CLpp)a, i. e. CMbCUabpbpa.

Dem.: $\quad G(1)$ MoCUabpb

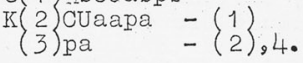

2 gives

8. (CLpLLp)a, i. e. CIbCUabpb KbCUab IcCUbcpc. Dem: : $\quad C(1)$ TibCUabpb

$$
\begin{aligned}
& \text { K(2) MbCUabIcCUbcUac - } 2 \\
& \mathrm{~K}\left\{\begin{array}{l}
3 \text { CUacpc } \\
4) \text { IbCUab IcCUb cpc }-\left(\begin{array}{l}
1 \\
2
\end{array}\right)
\end{array}\right.
\end{aligned}
$$

2, 5 give

$$
\text { 9. ( CMLpLp)a, }
$$

i.e. C乏bHUab пrcUbcpcitbCUabpb.

Dem.: C C $\quad$ bK $(1) \mathrm{Uab}$

$$
\begin{aligned}
& \text { 2\{ TcCUbcpc }
\end{aligned}
$$

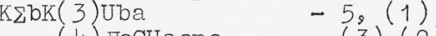

$$
\begin{aligned}
& \text { (4) McCUacpc } \\
& \text { K. (5) II cCUJa opa } \\
& \begin{array}{l}
-5,(1) \\
-(3),(2), 2 \\
=(4)
\end{array} \\
& \text { - (5) }
\end{aligned}
$$

Thus 1, or 4, gives T; 1, 2 or 4,2 gives $S 4 ; 1,3$ or 4,3 gives S5: and 1, 3 (but not 4,3 ) gives the equivalence of the above (IP) a with the usuaI S5( Lp)a, i. e ITapa.
Dem. :

$$
\begin{aligned}
& \text { K } 2 \text { II bCUbapb } \\
& \text { K } 3 \text { I: bpb }
\end{aligned}
$$$$
\begin{aligned}
& -(1), 5 \\
& =19(1),(2) \\
& -(3) .
\end{aligned}
$$

Fig. 1 Photograph of Prior's 1956 typescript. With thanks to the University of Canterbury's Library and Photographic Unit

modern eye it all looks very familiar, but also strange-no valuations assigning truth values, no metalanguage, no set-theoretic constructs offered as the semantic values of expressions of the object language.

The typescript carries the attribution 'C.A.M., August 1956; recorded and expanded A.N.P.'. It seems that what Prior 'expanded' was a brief note in Meredith's hand. I 
found this note among Prior's papers in the Bodleian Library, Oxford. It consists simply of eight lines of symbols - classic Meredith, who loved to compress his writing to the barest minimum. In the note, Meredith set down the reflexivity and transitivity axioms for ' $U$ ', the clauses for negation and implication, and the definition of L. He listed $\mathrm{K}, \mathrm{T}$ and $\mathrm{S} 4$ as theses, and he marked S5 with the reversed assertion sign used by Lukasiewicz to represent rejection.

The calculus set out in the 1956 typescript is purely formal and no philosophical interpretation is offered, either of the U-relation or of the nature of the objects to which the variables of the calculus refer. Once the formalism has been suitably explained, the Meredith-Prior semantics is a paradigm example of translational semantics. Meredith was in fact expounding a sophisticated theory of possible worlds in 1956 and earlier. ${ }^{29}$ But it seems that in 1956 neither he nor Prior had any interpretation to offer of the binary relation U. It was Geach who coincidentally suggested an interpretation, writing a letter to Prior in 1960 about 'jumping' between possible worlds. ${ }^{30}$ Prior seized on Geach's idea and took to describing $U$ as a relation of accessibility between worlds.

\section{The Smiley barrier}

As early as 1955, Timothy Smiley, at Cambridge, was pursuing an approach to modal semantics that he later referred to as translational semantics. ${ }^{31}$ Smiley's translational semantics has some of the same flavour as Prior's; Smiley's semantical predicate, linking sentences with their translations, is written 'may be read as', while (as mentioned previously) Prior used '[is] short for' in his 1962 article concerning world-accessibility.

Smiley appears to be the first person to have announced completeness proofs for the modal systems T, S4 and S5 relative to a semantics - a translational semantics - explicitly interpreted in terms of possible worlds; this was in $1957 .{ }^{32}$ The following is an extract from a 1957 lecture handout by Smiley setting out his semantics:

The operations ( )i are interpreted as translations of the descriptive or non-logical terms involved in the proposition, including an identity transformation $\mathrm{A}_{0}=\mathrm{A}$. Then in all of S, S4, S5, 1A may be read as (i) $\mathrm{A}_{\mathrm{i}}$ and $-1-\mathrm{A}$ as $(\mathrm{Ei}) \mathrm{A}_{\mathrm{i}}$ : that is, necessary truths are true in all 'possible worlds', and a proposition is possible if it is true in some 'possible world'. Choice between the three systems, or others, would be made on acceptance of the stipulations governing the translations ( )i - e.g. whether 'possible worlds' are to be arranged in a hierarchy of degrees of remoteness from the actual world or not. ${ }^{33}$

\footnotetext{
${ }^{29}$ Copeland (2006).

30 Geach's letter is reproduced in Copeland (2002, p. 119).

31 Letter from Smiley to Copeland (2000).

32 Copeland (2002, pp. 120-121).

33 Smiley (1957).
} 
Smiley later wrote regretfully:

It is a pity that [translational semantics] turns out to be a wrong turning outside the realm of propositional logic. ... Translational semantics is incapable of dealing with variations in the size of the domain. ${ }^{34}$

This is the 'Smiley barrier', the idea that translational semantics cannot be extended to quantificational logic in such a way that the domain of the quantifiers varies from possible world to possible world. However, Prior's type of translational semantics can in fact readily be extended to handle quantifiers and variable domains. The Smiley barrier is no real obstacle to translational semantics.

\section{The Barcan formula}

Ruth Barcan Marcus employed a notational variant of the formula (BF1), below, in her 1946 axiomatization of quantified modal logic. ${ }^{35}$ Instead of the notation used by Barcan, I shall continue to use Prior's L and M in order to express necessity and possibility. $\rightarrow$ expresses C. I. Lewis's strict conditional.

(BF1) $\mathrm{M} \exists x \mathrm{~F} x \rightarrow \exists x \mathrm{MF} x$.

(BF1) entails the material conditional:

(BF2) $\mathrm{M} \exists x \mathrm{~F} x \rightarrow \exists x \mathrm{MF} x$.

Both (BF1) and (BF2) are known in the literature as the 'Barcan formula'. Here the focus will be on (BF2); my remarks apply mutatis mutandis to (BF1).

Prior highlighted a previously unnoticed difficulty with the Barcan formula. ${ }^{36} \mathrm{He}$ used tenses to illustrate the problem, reading 'Mp' as 'It either is or has been or will be the case that p', and introducing a well-chosen illustration of quantifications involving varying domains: 'If it either is or has been or will be the case that someone is flying to the moon, then there is someone who either is flying or has flown or will fly to the moon'. Regarding this statement, Prior famously said:

And it is not easy to be quite happy about this. For suppose that in fact someone will fly to the moon some day, but not anyone who now exists. Then it will be true that it either is, has been, or will be the case that someone is flying to the moon; but it will not be true that there is someone who either is flying or has flown or will fly to the moon. ${ }^{37}$

Re-expressing this idea without equating Mp to $\mathrm{p} \vee \mathrm{Pp} \vee \mathrm{Fp}$, Prior's point is simply this: one cannot in general infer that there exists someone who will possibly reach the moon from the proposition that possibly someone will reach the moon.

\footnotetext{
34 Letter from Smiley to Copeland (2000).

35 Marcus (1946).

36 Chap. 2 of Williamson (2013) gives an excellent history of the Barcan formula.

37 Prior (1957a, p. 26).
} 
The Barcan formula serves as a touchstone when developing a modal semantics in which the domains of quantification vary. When the domain is constant, the semantics should validate the Barcan formula; and the semantics should also validate the Barcan formula where the domains of the two existential quantifiers are distinct but overlap appropriately; but where the domains do not overlap, the semantics should not validate the formula, for the reason that Prior gave.

Barcan Marcus recommended using a substitutional interpretation of the quantifiers when giving a semantics for quantified modal logic, and I shall follow her suggestion here. ${ }^{38}$ In the following extension of Prior's modal semantics to quantified modal logic, a substitution class $\mathrm{T}_{w}$ is associated with each possible world $w .{ }^{39} \mathrm{~T}_{w}$ contains all atomic singular terms that denote in $w$, and the atomic singular terms are assumed to denote rigidly (that is, an atomic term never has different denotations in different worlds; although it may fail to denote anything in some worlds). Each existentially quantified formula is translated by a sentence in which the formula, say $\exists x \mathrm{~F} x$, is replaced by a substitution instance of the formula, say $\mathrm{F} t$, where $t$ is a member of the substitution class associated with the world in question. Since the substitution class contains only terms that denote (in that world), quantification remains tied to existence; and variations in the substitution classes from world to world are tied to, and represent, variations in the domain of quantification.

The following two translational clauses $(\exists)$ and $(\forall)$ extend Prior's semantics to quantified modal logic. For clarity, $\Sigma$ is used for the existential quantifier of the semantical language, and $\Pi$ for the universal quantifier:

$$
\begin{aligned}
& (\exists)(\exists x \mathrm{~F} x) w=\Sigma t\left(t \in \mathrm{T}_{w} \&(\mathrm{~F} t) w\right) \\
& (\forall)(\forall x \mathrm{~F} x) w=\Pi t\left(t \in \mathrm{T}_{w} \rightarrow(\mathrm{F} t) w\right)
\end{aligned}
$$

The following derivation illustrates the semantics. The derivation establishes the Barcan formula when the substitution classes invoked in the two applications of $(\exists), \mathrm{T}_{a}$ and $\mathrm{T}_{b}$, are identical. A minor variation of the derivation also establishes the formula when $\mathrm{T}_{b} \subseteq \mathrm{T}_{a}$.

$$
\begin{aligned}
(\mathrm{M} \exists x \mathrm{~F} x) a & =\Sigma b(\mathrm{U} a b \&(\exists x \mathrm{~F} x) b) \\
& =\Sigma b\left(\mathrm{U} a b \& \Sigma t\left(t \in \mathrm{T}_{b} \&(\mathrm{~F} t) b\right)\right) \\
& =\Sigma b\left(\mathrm{U} a b \& \Sigma t\left(t \in \mathrm{T}_{a} \&(\mathrm{~F} t) b\right)\right) \\
& =\Sigma t\left(t \in \mathrm{T}_{a} \& \Sigma b(\mathrm{U} a b \&(\mathrm{~F} t) b)\right) \\
& =\Sigma t\left(t \in \mathrm{T}_{a} \&(\mathrm{MF} t) a\right) \\
& =(\exists x \mathrm{MF} x) a
\end{aligned}
$$

Prior's counterexample to the Barcan formula is formalized very naturally in this semantics. Assume for simplicity that there are only two possible worlds, $a$ and $b$,

\footnotetext{
38 Marcus (1962). See also Copeland (1982, 1985).

39 As in my 1982 and 1985, although the focus there was on truth-conditional semantics, not translational semantics.
} 
where Uab. Again for simplicity, let us follow Saul Kripke's suggestion that it is natural to assume that an atomic predicate should be false in a world of all those individuals not existing in that world' ${ }^{40}$ The counterexample can, however, equally well be formalized in a version of the semantics that deems atomic sentences truthvalueless in worlds in which a contained atomic singular term fails to denote (in the spirit of Prior's System Q). ${ }^{41}$ Let $\mathrm{T}_{a}$ and $\mathrm{T}_{b}$ be distinct singletons, $\{s\}$ and $\{u\}$, such that $(\mathrm{F} u) b$ but not (Fs) $a$ (and not ( Fs) $b$, as $s$ does not denote anything at $b$ ). Since $(\exists x \mathrm{~F} x) b$, it follows that ( $\mathrm{M} \exists x \mathrm{Fx}) a$; but since it is not the case that (MFs) $a$, neither is it the case that $(\exists x \mathrm{MF} x) a$.

In conclusion, Priorean translational semantics escapes the Smiley barrier when the quantifiers are treated substitutionally; and moreover, Prior's translational approach to modal and temporal semantics is far from being the lame duck that it might have appeared to be, in the wake of the Tarskian revolution.

\section{References}

Copeland, B. J. (1979). On when a semantics is not a semantics. Journal of Philosophical Logic, 8, 399-413. Copeland, B. J. (1982). A note on the Barcan formula and substitutional quantification. Logique et Analyse, 97, 83-86.

Copeland, B. J. (1985). Substitutional quantification and existence. Analysis, 45, 1-4.

Copeland, B. J. (1986). What is a semantics for classical negation? Mind, XCV, 478-490.

Copeland, B. J. (1996). Prior, Arthur. Stanford encyclopedia of philosophy. http://plato.stanford.edu/entries/ prior/.

Copeland, B. J. (2002). The genesis of possible worlds semantics. Journal of Philosophical Logic, 31, 99-137.

Copeland, B. J. (2006). Meredith, Prior, and the history of possible worlds semantics. Synthese, 150, 373397.

Cresswell, M. J. (2015). Prior on the semantics of modal and tense logic. Synthese. doi:10.1007/ s11229-015-0949-0.

Davidson, D. (1967). Truth and meaning. Synthese, 17, 304-323.

Drake, F. R. (1962). On McKinsey's syntactical characterizations of systems of modal logic. Journal of Symbolic Logic, 27, 400-406.

Evans, G., \& MacDowell, J. (Eds.). (1976). Truth and meaning: Essays in semantics. Oxford: Oxford University Press.

Fodor, J. A. (1975). The language of thought. New York: Crowell.

Kripke, S. A. (1963). Semantical considerations on modal logic. Acta Filosophica Fennica, 16, 63-72.

Lewis, D. K. (1970). General semantics. Synthese, 22, 18-67.

Marcus, R. B. (1946). A functional calculus of the first order based on strict implication. Journal of Symbolic Logic, 11, 1-16.

Marcus, R. B. (1962). Interpreting quantification. Inquiry, 5, 252-259.

Meredith, C. A., \& Prior, A. N. (1956). Interpretations of different modal logics in the 'Property Calculus'. Mimeograph, University of Canterbury Philosophy Department. Reprinted in Logic and reality: Essays on the legacy of Arthur Prior, by B. J. Copeland, (Ed.), 1996, Oxford: Clarendon Press.

Prior, A. N. (1957a). Time and modality. Oxford: Oxford University Press.

Prior, A. N. (1957b). Critical notice of Alfred Tarski, Logic, semantics and metamathematics. Mind, 66, 401-410.

Prior, A. N. (1958). The syntax of time-distinctions. Franciscan Studies, 18, 105-120.

Prior, A. N. (1961). On a family of paradoxes. Notre Dame Journal of Formal Logic, 2, 16-32.

\footnotetext{
40 Kripke (1963, p. 66).

41 Prior (1957a, Chap. 5). See my 1982 details of the revised counterexample (although the treatment there is model-theoretic, not translational).
} 
Prior, A. N. (1962a). Possible worlds. Philosophical Quarterly, 12, 36-43.

Prior, A. N. (1962b). Tense logic and the continuity of time. Studia Logica, 13, 133-148.

Prior, A. N. (1976). What is logic? In A. N. Prior, Papers in logic and ethics. London: Duckworth.

Smiley, T. J. (1957). Modal logic. Lecture handout, Department of Philosophy, University of Cambridge. Williamson, T. (2013). Modal logic as metaphysics. Oxford: Oxford University Press. 